\title{
Mercury stable isotope fractionation during aqueous photoreduction in sulfidic environment
}

\author{
YAQIU ZHAO AND WANG ZHENG
}

Tianjin University

Presenting Author: yaqiu_77@tju.edu.cn

Mercury $(\mathrm{Hg})$ is a global pollutant with complex transformations in the environment. The fractionation of $\mathrm{Hg}$ stable isotopes has been widely used as a powerful tracer for $\mathrm{Hg}$ cycling, for that it exhibits unique mass independent fractionation (MIF), particularly during photochemical reactions. The aqueous photoreduaction of various $\mathrm{Hg}$ (II) speices is a critical process controlling $\mathrm{Hg}$ cycles and one of the major processes that generate MIF. However, the mechanism of $\mathrm{Hg}$ MIF during photoreduction has not been fully understood. Previous studies on $\mathrm{Hg}$ isotope fractionation during photoreduction have mostly focused on oxic aquatic environment where $\mathrm{Hg}(\mathrm{II})$ is dominantly complexed by organic matter, but there is no systematic study in sulfidic water, where $\mathrm{Hg}$ (II) is complexed by sulfide. Previous studies have shown that the sign and magnitude of $\mathrm{Hg}$ MIF are sensitive to the type of ligands and dissolved oxygen in solution. Thus $\mathrm{Hg}$ MIF has the potential to act as a proxy of ocean redox changes. We have demonstrated that Hg MIF in black shale can record photic zone euxinia (PZE, shallow anoxic and sufide rich condition in ancient ocean), which may have played a critical role in mass extinction and the evolution of life. Here we experimentally investigate $\mathrm{Hg}$ isotope fractionation during photoreduction of $\mathrm{Hg}(\mathrm{II})$ in sulfide-rich water, which is representative of PZE in ancient ocean and in some anoxic coatsal settings in modern environment. Our goals were to further understand the mechanism of $\mathrm{Hg}$ MIF in its photochemistry, and develop the use of $\mathrm{Hg}$ MIF as a paleoredox proxy.

We observed both mass dependent fractionation with enrichment of heavier isotopes in the reactant $\mathrm{Hg}$ (II) and significant negative MIF that caused depletion of odd mass isotopes in $\mathrm{Hg}(\mathrm{II})$. This negative direction is opposite to the positive MIF previously observed in photoreduction in oxic waters, but is similar to the MIF during photoreduction of $\mathrm{Hg}$ (II) complexed by reduced organic thiol. More details of the experiment are still in progress to investigate the effects of $\mathrm{Hg}$ speciation, and irradiation wavelength and other environment parmeters on $\mathrm{Hg}$ MIF. 